\title{
Study of morphology wear model of moulds from alloys of aluminium EN AW-6082 in injection process.
}

\author{
${ }^{1}$ Pereira, $A^{\mathrm{a}}$.; ${ }^{2}$ Hernández, P.. ; ${ }^{1}$ Martínez J.; ${ }^{1}$ Pérez, J.A.; ${ }^{3}$ Mathia, T.. \\ ${ }^{1}$ Faculty of Industrial Engineering, University of Vigo, Campus Marcosende, Vigo, 36210, Spain \\ ${ }^{2} 2$ Hergome Ltd. (Metal Machining ) Ctra. Tameiga a Mos n0149 / Nave 5 - 36416, Spain \\ ${ }^{3}$ Ecole Centrale de Lyon, Laboratoire de Tribologie et Dynamique des Systèmes, Ecully, France \\ apereira@uvigo.es, ${ }^{\mathrm{b}}$ phernandez@hergome.com, ${ }^{\mathrm{c}}$ Thomas.Mathia@ec-lyon.fr
}

Keywords: Roughness, surface topography, mould wear, polymer injection process.

\begin{abstract}
The main objective of this research is study the wear of a mould, made in easy machining material; aluminium EN AW-6082 T4 and the surface characterization of mould cavity, used in a polymeric manufacturing process, after several injection cycles. To evaluate the effectiveness of this materials in the plastic injection process, a test part with different thicknesses and shapes was designed and some inserts of aluminium were made. In the way of plastic material, a $30 \%$ fiberglass reinforced polyamide PA6 has been employed in the experimental procedure. A reusable injection mould was used and surface measurements of mould cavity have been performed after 9200 cycles. The surface topography has been measured by using optical interferometry profiling technology and there have also been obtained roughness and surface parameters according to ISO 25178 and EUR15178N. In order to surface characterization, different areas of the mould cavity with different geometries, has been analysed. The behaviour of wear rate has been analysed by the study of the evolution of the surface parameters and functional parameters in different points of the surface mould.
\end{abstract}

\section{Introduction}

In order to cope with flexibility and short time for new product development, mould industry has made use of soft materials for cavity inserts in injection mould. However, one of the main problems using this kind of material is related to its wear rate. This paper provides a contribution for measuring the topographic wear in injection moulds cavities.

One type of material used in automotive parts industry is glass fibre reinforced plastics. These kinds of materials are extremely abrasive for mould cavities. Silva et al studied behaviour of TiAlSiN and CrN/CrCN/DLC sputtered coatings in steel moulds to determine the one with better wear resistance for plastic injection processes using glass fibers as reinforcement. [1]

More specifically, Zhong, Z.W. et al has studied the behaviour of a rapidly solidified aluminium alloy compared with beryllium copper and 6061 aluminium alloys in terms of their wear rates, hardness and performance as mould insert materials, for moulding a plastic lens with spherical surfaces. [2]

Various fibre orientation patterns in the melt may arise in processing filled polymers. There are a number of distinct layers within the moulding with different fibre alignments [3]. In the skin layer, the fibre orientation is predominantly parallel to the flow direction due to the elongation forces developing during flow at the melt front as well as due to the shear flow after the front has passed. In contrast, a random-in-plane alignment of fibres is observed in the core layer due to slower cooling rate and lower shearing. Exactly this mutual relative motion of self-orienting filler and mold wall causes adhesive and abrasive wear of injection moulds. Wear intensity is affected predominantly by the kind of processed polymer, moulding shape and dimensional complexity, its segmentation and required precision, and the temperature and pressure of the injected polymer [4] [5].

Bergstrom et al study the behaviour of three die tool steels for plastic dies, ranging from a fully martensitic ingot cast medium hardness steel to a high hardness powder metallurgical steel. This measurement of wear rate and correct tribologic test method is difficult to achieve, due to the 
complexity of the forming technology and the large different shapes of used cavity [6]. This motivated the present study where the severe conditions of process such as velocity of polymer and high pressure of the viscoelastic material had been needed to fill the cavity.

This work emphasizes on the method of measuring the aluminium mould wear rate in order to study the different mechanism of wear that occurs in the cavity when a glass fibre reinforced polyamide had been injected. To enable economic improvement there is a need to both increase the die tool wear resistance and to improve the predictability of it.

\section{Methodology \\ Materials}

The mould materials used for this study are aluminium alloy EN AW-6082 T4 (Al Mg Si 1). The physical properties and alloy components are indicated in Table 1. These materials are commonly used in the industry for manufacturing injection of small series or prototypes.

As plastic material injected, this work has used polyamide 6 reinforced with $30 \%$ glass fibre (Dilamid ${ }^{\circledR} 6$ FV Black 3136), with a density of $1.36 \mathrm{gr} / \mathrm{cm} 3$, was chosen. The characteristics of this material are illustrated in Table 2 . To avoid the hygroscopic effect, this material was subjected to drying at the temperature of $80{ }^{\circ} \mathrm{C}$ for 5 hours.

Table 1: Aluminium alloy

\begin{tabular}{|c|c|}
\hline & $6082 \mathrm{~T} 4$ \\
\hline $\begin{array}{l}\text { Hardness Brinell } \\
\text { (HBW 2,5/31,5 ) }\end{array}$ & 82 \\
\hline Density $\left[\mathrm{g} / \mathrm{cm}^{3}\right]$ & 2.71 \\
\hline Yield strength $\left[\mathrm{N} / \mathrm{mm}^{2}\right]$ & 260 \\
\hline Tensile Strength $\left[\mathrm{N} / \mathrm{mm}^{2}\right]$ & 340 \\
\hline Thermal cond. [W/km] & 170 \\
\hline
\end{tabular}

Table 2: Dilamid 6FV Black

\begin{tabular}{|l|r|}
\hline & Dilamid® 6 FV Black 3136 \\
\hline Density (ISO 1183)[g/cm $\left.{ }^{3}\right]$ & 1,36 \\
\hline Hardness, Rockwell R & 121 \\
\hline Tensile Strength at Break [Pa] & $159 \times 10^{6}$ \\
\hline Tensile Strength, Ultimate [Pa] & $155 \times 10^{6}$ \\
\hline Molding Shrinkage & $0,30 \%$ \\
\hline Melting Point [ $\left.{ }^{\circ} \mathrm{C}\right]$ & 220 \\
\hline Melt Temperature $\left[{ }^{\circ} \mathrm{C}\right]$ & $255-275$ \\
\hline Mold Temperature [ $\left.{ }^{\circ} \mathrm{C}\right]$ & $70-110$ \\
\hline Drying time [ hours] & $2-8$ \\
\hline
\end{tabular}

Design of part, mould cavity and insert pins.

To study the surface characteristics of the mould after a number of injection cycles, a experimental geometry was designed in platform Catia V5 (Figure 1 ) in order to allow the study of the behaviour wear at different points. Thus, the different mechanism of wear can be compared in different points/surfaces of the mould. The concept of Reusable Injection Mould (RIM) formed by a "structure" completely reusable and a "interchangeable ensemble" specially designed for each project, was used on this work [7] [8].

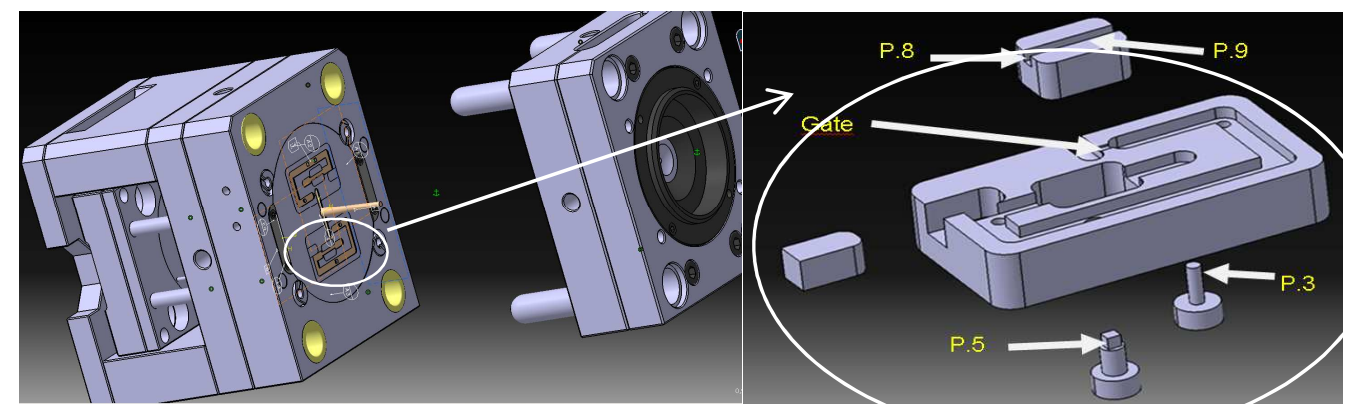

Figure 1: Design of niìold

\section{Injection Moulding Process}

An injection moulding machine ENGEL Victory 200/28 without columns was used for the injection process. The conditions of process was shown in Table 3 
Table 3: Injection conditions.

\begin{tabular}{|l|r|}
\hline \multicolumn{2}{|l|}{ Parameters injection process } \\
\hline PA6 Volume $\left[\mathrm{m}^{3}\right]$ & $2,8 \times 10^{-5}$ \\
\hline Injection speed $\left[\mathrm{m}^{3} / \mathrm{s}\right]$ & $11 \times 10^{-5}$ \\
\hline Cooling time $[\mathrm{s}]$ & 3 \\
\hline Holding pressure $[\mathrm{Pa}]$ & $29-0 \times 10^{6}$ \\
\hline Pressure injection $[\mathrm{Pa}]$ & $22 \times 10^{6}$ \\
\hline $\begin{array}{l}\text { Temperature cylinder }\left[{ }^{\circ} \mathrm{C}\right] \\
\text { (zones } 1,2,3 \text { and } 4)\end{array}$ & $285 ; 280 ; 275 ; 270$ \\
\hline
\end{tabular}

\section{Measurements.}

To characterize the roughness at different locations of the cavity, according to the defined areas in the part, a non-contact optical profilometry measurement tool (Wyko NT-1100) was used [9]. The measurements were carried out on aluminium alloy for selected cycles of injection at 200, 800, 2,000, 5,600, and 9,200 injection cycles. In this work, measurements were taken at marked points on the inserts (Fig.2 ) prepared by milling process.

In this research was analysed next points:

Point 3 (P3): Frontal impact within cylinder shape

Point 5 (P5): Frontal impact within prismatic shape

Point 8 (P8): For study centrifugal forces and frontal impact

Point 9 (P9): Narrowing area to study the increase of fiber speed.

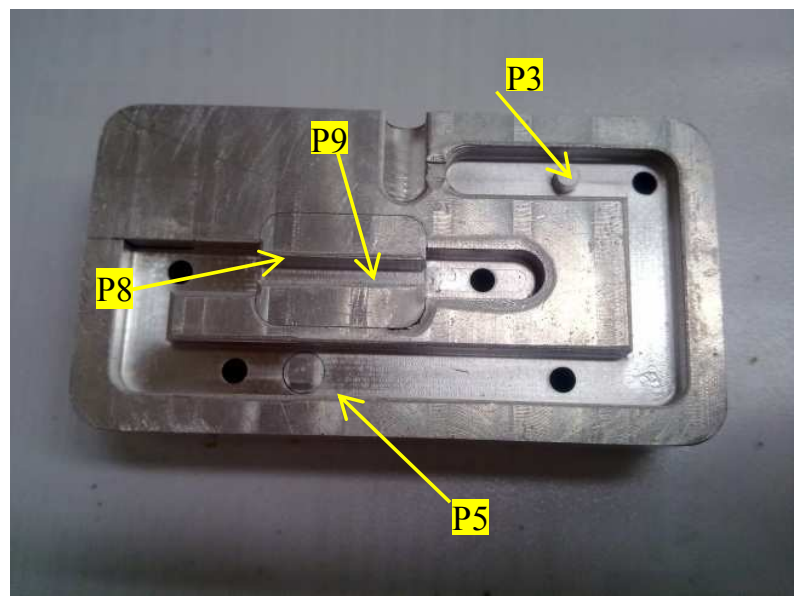

Figure 2: Points of measurement.

For all this chosen experimental points, the special roughness parameters such as $\boldsymbol{S}_{\boldsymbol{s} k}, \boldsymbol{S}_{\boldsymbol{k} u \text {, }}$ mentioned in ISO25178, and $S_{b i}$ according to EUR $15178 \mathrm{~N}$ were obtained and calculated with the temporal license of software Mountain Map [10]

Table 4: Design of Experiments

\begin{tabular}{|l|c|l|c|c|}
\hline Polym. & $\begin{array}{c}\text { Material } \\
\text { Mold }\end{array}$ & Cycles & Points of measurement & $\begin{array}{c}\text { Surface } \\
\text { Parameters }\end{array}$ \\
\hline & & 200 & Point 3 & \\
PA 6.6 30\%FG & 6082 T4 & $\mathbf{8 0 0}$ & Point 5 & $S_{\boldsymbol{s} \text {, }}$ \\
& & 5.600 & Point 8 & $\boldsymbol{S}_{\boldsymbol{k} u}$ \\
& & 9.200 & Point 9 & $\boldsymbol{S}_{\boldsymbol{b} \boldsymbol{v}}$ \\
& & & \\
\hline
\end{tabular}




\section{Results}

Simulation of the tangential and frontal flow of glass fibre was analysed in MoldFlow ${ }^{\circledR}$ at Point 3 (cylindrical obstacle), Point 5 (rectangular obstacle), Point 8 (area reduction) and Point 9 that contain a narrowing, to study the influence of the increase of the fibre speed. Figure 3 shows material flow representation for this mentioned zones.

It can be seen in figure 3 that at different points occur significant speed variations resulting different wear mechanisms. There is a frontal impact of glass fibres at point 5, at point 8 and in the central area of point 3. As the simulation, this frontal impact results in a decrease of speed, with values of $3.32 \mathrm{~cm} / \mathrm{s}$ at point $5,7.3 \mathrm{~cm} / \mathrm{s}$ in point 8 and $16.98 \mathrm{~cm} / \mathrm{s}$ in central point 3 . However in point 9 with an average speed of $30.78 \mathrm{~cm} / \mathrm{s}$ the position of the longitudinal fibres is in the direction of flow and in this case there is tangential contact.

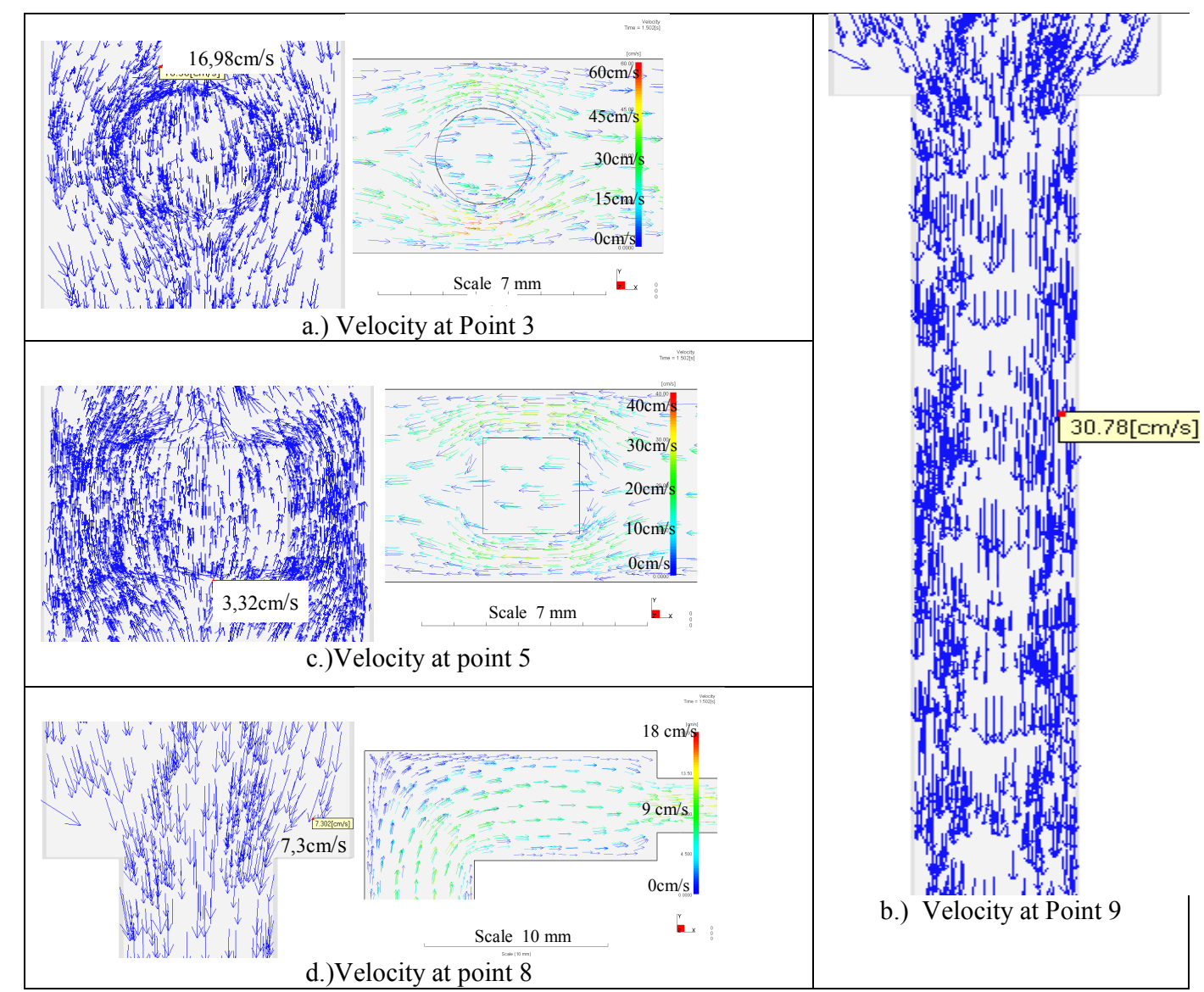

Figure 3: Simulation of material flow velocity

The simulated conditions filling rate of the polymeric material doesn't change along injection cycles. As it can be observed in the figure 4, the worn surface corresponds to Point 5 and point 8 . In these areas the glass fiber endings seem to either leave traces of indentations and make an effect of micro cutting.
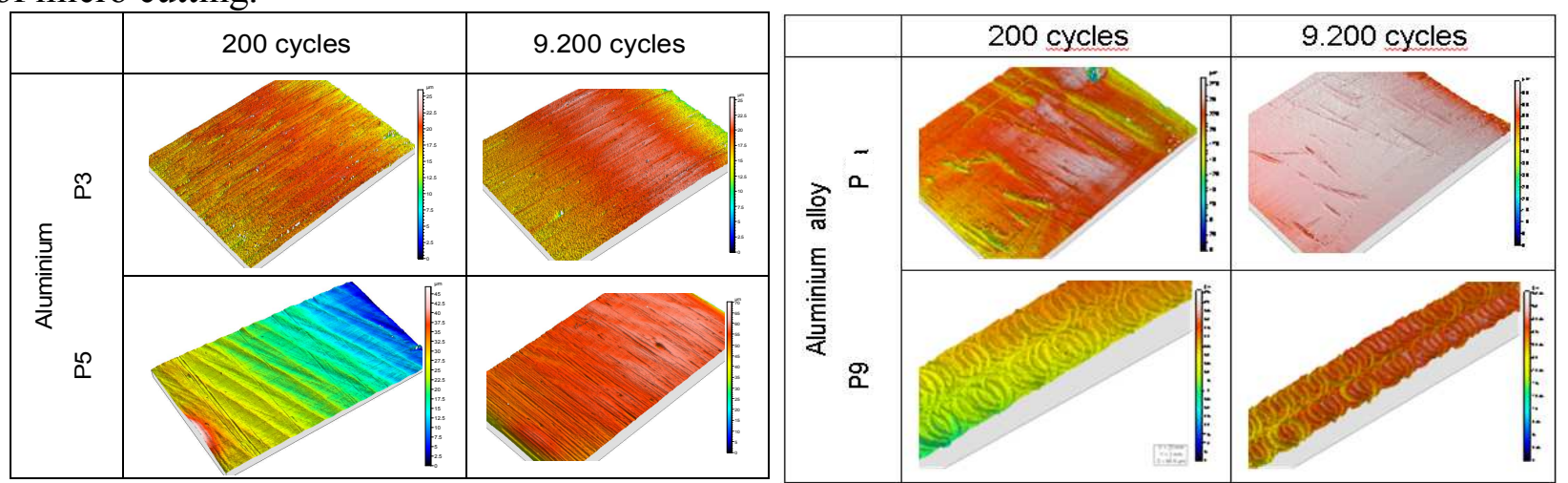

Figure 4: Topography images of Aluminium Alloy 
In the wear process, the surface skewness parameter $S s k$, is one of the most important, due to the effect of the truncation of asperities [11]. As a consequence of the abrasive wear, the proportion of valleys over peaks increases, resulting in a lower $S s k$ value.

In the figure 5 , it can be represented the parameter $S_{S k}$ versus the number of cycles. It can be observed that there are important changes of the initial topography from 200 to 800 cycles, due to an intensive wear.

It also shows that the point 5 , has a lower $S_{S k}$ parameter at 9200 cycle due to increased abrasive wear. Point 8 also has a significant reduction in this parameter from Ssk $=-1.13$ to Ssk $=-2.04$.

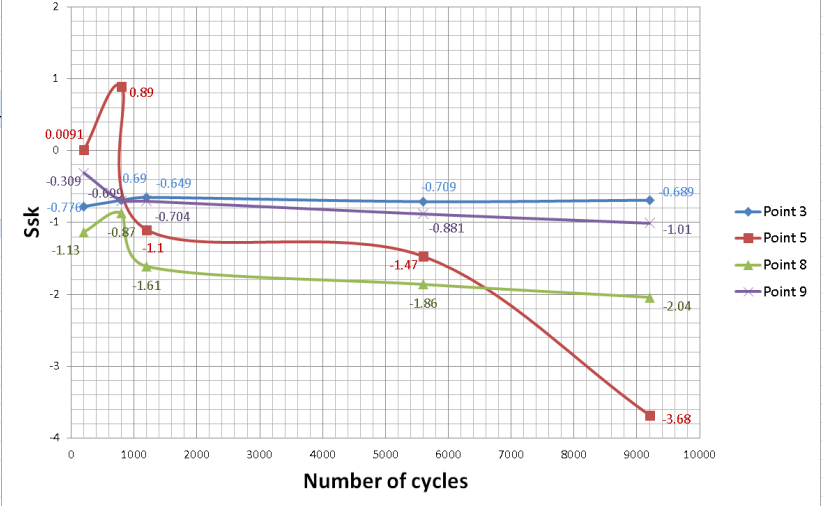

Figure 5: Ssk versus cycles

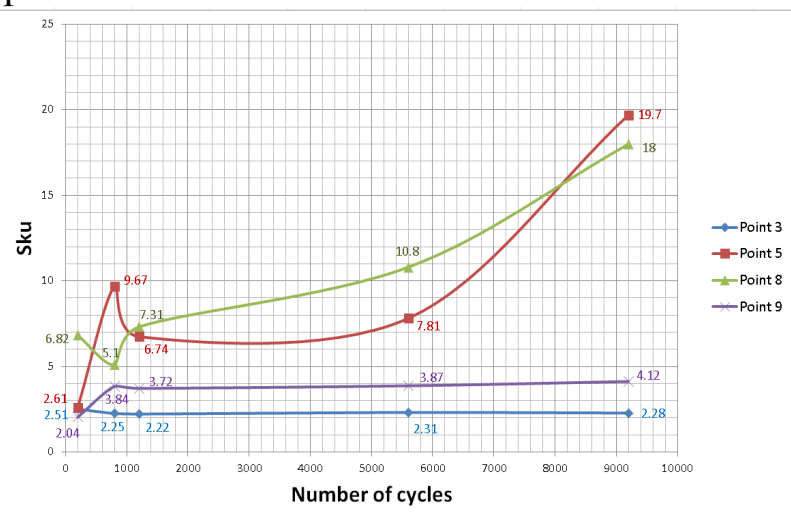

Figure 6: $S k u$ versus cycles

The surface kurtosis, Sku, describes the "peakedness" of the surface topography. This parameter is sensitive to deep grooves and high peaks in surface topography. The fourth power in definition of $S k u$ explains this phenomenon. The Figure 6 shows the evolution of $S k u$ versus the cycles in the analysed points. The highest value of $S k u$, correspond to point 5, with a value of $S k u=19.7$ follow by the point 8 with $S k u=18$. In the figure 7 it can be shown the relation $S k u$ versus $S s k$ in points 5 and 8.

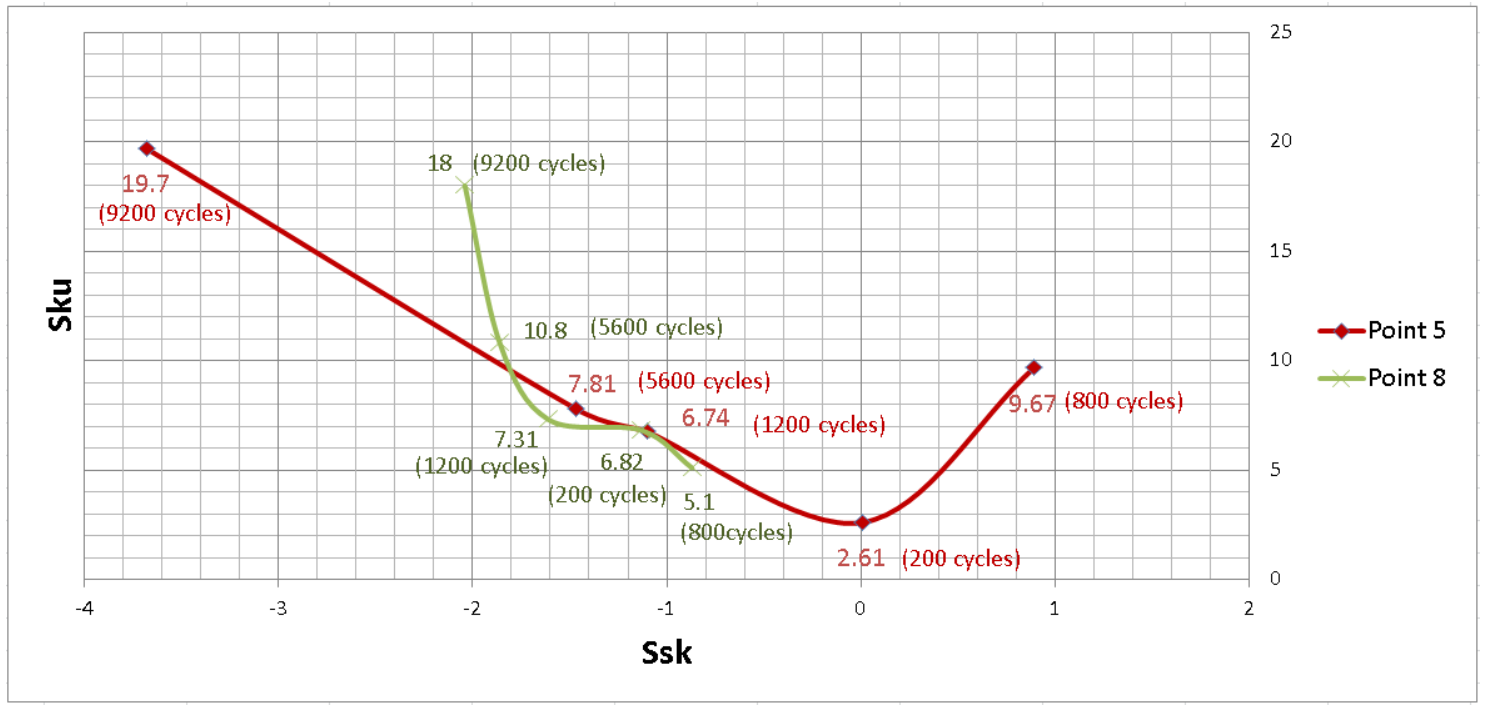

Figure 7: Sku versus $S s k$

\section{Conclusions}

From the experimental results and analysis of the wear in different points of the mould, the next conclusions can be obtained:

- There are an initial intensive wear between 200 and 800 cycles, in all the points, particularly in areas of frontal impact of glass fibres.

- The abrasive effect of the glass fibres of the flow is more intensive in frontal impact than in the longitudinal impact of flow.

- Wear rate of mould increase due to the frontal obstacles. 
- The parameters $S s k$ decrease with the increment of the wear rate.

- The parameter $S k u$ increase with the increment of the wear rate.

The authors wish to thank to Ministry of Science and Innovation of Spain by its support under the grant DPI2008-06342/DPI and to Digital Surf ${ }^{\circledR}$ for letting this research group experiment with a temporal license of their software Mountains Map 6.2.

\section{References}

[1] Silva, F.J.G.; Martinho, R.P.; Alexandre, R.J.D.; Baptista, A.P., "Increasing the wear resistance of molds for injection of glass fiber reinforced plastics," Wear, pp. 2494-2499, 2011.

[2] Zhong, Z.W.; Leong, M.H.; Liu, X.D., "The wear rates and performance of three mold insert materials," Materials and Design, vol. 32, p. 643-648, 2011.

[3] Brezinova, J; Guzanova, A., "Friction Conditions during the Wear of Injection Mold Functional Parts in Contact with Polymer Composites," Journal of Reinforced Plastics and Composites, 2010.

[4] Franklin, S. E., "Wear Experiments with Selected Engineering Polymers and Polymer Composites under Dry Reciprocating Sliding Conditions," Wear,, 2001.

[5] Michael, P. C., Rabinowicz, E. and Iwasa, Y., "Friction and Wear of Polymeric Materials," Cryogenics, 1991.

[6] Bergstrom, J.; Thuvander, F.; Devosb, P.; Boher, "Wear of die materials in full scale plastic injection moulding of glass fibre reinforced polycarbonate," Wear, vol. 251, p. 1511-1521, 2001.

[7] Hernández, P.; Ares, E.; de la Mano, R.; Pereira, A., "Characterization of wear during the injection process," in PMI 2010, Gante, 2010.

[8] Pousada, A. S.; Hernández, P.; Ares, E., "New trends on the design and manufacture of injection moulds, reusability and recycling," in Proceeding PMI 2005 Conf, Gent, Belgium, April 2005, p. aer555.

[9] Mathia, T.G.; Pawlus, P.; Wieczorowski, M, "Recent trends in surface metrology," Wear, p. 494-508, 2011.

[10] Blateyron, F. "New 3D parameters and filtration techniques," Digital Surf, 2006.

[11] Jenga, Y.; Gao, C., "Changes of Surface Topography during Wear for Surfaces with Different Height Distributions," Tribology Transactions, vol. Volume 43, 2000. 